Theme: Cardiology

Keywords: Adolescents, children, quality of life, PedsQL Cardiac Module, validation.

Introduction: Measuring quality of life (QoL) in children and adolescents with congenital heart disease $(\mathrm{CHD})$ is of great clinical importance.

Objective: The aim of the study was: (a) to adapt the PedsQL Cardiac Module for children aged two to 18 years with $\mathrm{CHD}$ in a sample of the Greek population; (b) to determine its reliability and validity.

Methods: Forward and backward translation methodology was used. Parents and children completed the instrument during: (a) hospitalization and (b) visits in the paediatric cardiology outpatient department. Cross-informant variance between children and parents was thoroughly assessed.

Results: Missing item responses did not exceed 5\%. All internal consistency reliability coefficients for the inventory exceeded the minimum standards for group comparisons, over 0.75 . Hypothesized correlations between cardiac module and core scales were statistically significant, $(p<0.05)$. Agreement between children and parents was relatively high. Pilot study results will be additionally presented.

Conclusion: The findings support the feasibility, reliability and validity of the Greek translation of the PedsQL Cardiac Module in children with congenital heart defect (CHD).

\title{
OC20 - Skin-to-skin contact in the first hour of life
}

\author{
Manuela Ferreira (Portugal)i; Tânia Vaz (Portugal)2; \\ Graça Aparício (Portugal)ํㅜ; João Duarte (Portugal) ${ }^{1}$
}

\section{${ }^{1}$ Instituto Politécnico de Viseu - Escola Superior de Saúde CI\&DETS; ${ }^{2}$ Centro Hospitalar Cova da Beira}

Theme: Child protection and managing risk.

Keywords: Breastfeeding, newborn, skin-to-skin contact.

Background: Skin-to-skin contact in the first hour of life has benefits for the mother and the newborn, as well as a major role in establishing breastfeeding.

Objectives: Determine the prevalence of skin-to-skin contact and breastfeeding within the first hour of life.

Method: A systematic review of literature followed by a quantitative and simple descriptive cross-sectional study, according to a non-probability analyzing of 382 clinic records of postpartum mothers.

Results: Evidence that early skin-to-skin contact immediately after birth is a potential sensory stimulus, which covers the newborn warming, tactile and active stimulation, respiratory rates and level of blood glucose, reduces baby crying and promotes breastfeeding. In the sample, about $92.6 \%$ of the mothers put the baby to the breast in the first hour of life, but only $26.6 \%$ made skin-to-skin contact with the baby.

Conclusion: Despite the scientific evidence of the benefits of skin-to-skin contact 


\title{
OC21 - Psychosocial functioning of parents of infants diagnosed with complex congenital heart defect
}

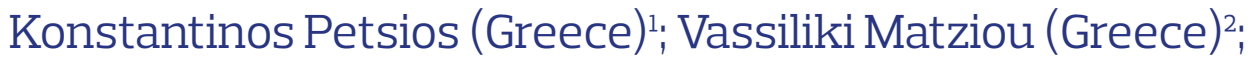 \\ Ioanna Voutoufianaki (Greece) ${ }^{1}$; Anna Manatou (Greece)ㄹ; \\ Prodromos Azariadis (Greece) ${ }^{1}$; Spiros Rammos (Greece) ${ }^{1}$ \\ ${ }^{1}$ 'Onassis' Cardiac Center; ${ }^{2}$ - Faculty of Nursing, National and Kapodistrian \\ University of Athens
}

Theme: Parenting/parenthood.

Keywords: Congenital heart defect (CHD), parenting, psychosocial.

Introduction: The diagnosis of complex CHD impacts the psychosocial status of parents and their functioning.

Purpose: A critical evaluation of the literature concerning the psychosocial parental response to their infant's diagnosis of complex CHD.

Methods: Systematic review of 18 articles published after 2000 in PubMed and CINAHL.

Results: The impact of an infant's CHD on the family functioning is determined both by child's medical condition and family's psychosocial factors. The majority of parents experience intense loss and numerous stressors. Nurses and physicians need to be sensitive to the needs, thoughts and experiences of the parents when discussing treatment options. Families with poor social support networks may have the greatest need for professional interventions.

Conclusions: Appropriate interventions assist the majority of parents to adapt to the diagnosis of complex CHD. However, there are a number of parents with an increased psychosocial risk associated with higher rates of emotional distress.

\section{OC22 - Light House Project}

Gonçalo Pereira (Portugal)i; Ana Baridó (Portugal)²

${ }^{1}$ Universidade Católica Portuguesa - Instituto de Ciências da Saúde - Lisboa;
${ }^{2}$ ACES Leiria

Theme: Accreditation and quality improvement.

Keywords: Adolescents, indicators, nursing care, nursing consultation, quality improvement.

In the context of primary health care internship of a master's degree in paediatric nursing ministered by 'Universidade Católica Portuguesa' in Lisbon, and after diagnosing a 